\title{
A Study on Fashion Overcoats in the Republic of China*
}

\author{
Zhaoqing Li \\ Fuzhou University \\ Fuzhou, China
}

\begin{abstract}
Fashion overcoats came in vogue in Shanghai in the Republic of China (ROC) (1912-1949) in the mid \& late $1920 \mathrm{~s}$, before became the trendy wear for modern ladies of numerous big cities of the country. Fashion overcoats in the Republic of China featured a distinct law of changes, with decade-identifiable design elements such as fits, fabrics, and colors. Fashion overcoat was one of the most representative lady fashion wears in the Republic of China time. With the sophisticated craftsmanship, exquisite materials, and elegant design, it reflected the highest level in fashion wear of that time.
\end{abstract}

\section{Keywords-Fashion overcoats; ROC; Shanghai}

\section{INTRODUCTION}

Fashion overcoat in the Republic of China was the essence of fashion of modern China, and was a vivid reflection of socio-cultural diversity of that time. With its fruitful achievement and high attainments in the exploration for modernization of traditional Chinese dress, it offered a great lesson to learn for contemporary fashion design in China.

\section{THE ORIGIN OF THE FASHION OVERCOATS OF THE REPUBLIC OF CHINA}

Velvet overcoat was the first of its kind to came in vogue in ROC (1912-1949) in cloak's stead to protect against the coldness outdoors. After that, varieties, styles and fabrics of overcoat become more and more diverse. To list by season, there were winter overcoat, lined overcoat for Spring and Autumn, unlined overcoat for late Spring and early Autumn, and silk overcoat outside cheongsam to add to beauty and harmonize colors at Summer night.

Shanghai was the center of fashion at the ROC time, with lady overcoat taking the biggest share in tailoring and sales. Even ladies in other parts of China took it as a pride to wear overcoats bought in Shanghai. With the spread and popularization of fashion, other economically and culturally developed cities like Beijing, Tianjin, Guangzhou, Nanjing also had a more and more abundant supply of fashion overcoats, becoming important cities for fashion overcoats of the time.

* Social Science Planning Fund Program in Fujian Province (No:FJ2016C194)

\section{THE LAW OF CHANGES FOR FASHION OVERCOAT IN ROC}

\section{A. The 1920s}

Nylon overcoat came into fashion in Shanghai in the 1920s. Around 1926, it was widely accepted as a substitution for cloak, lined cheongsam, and lined skirt and dress in cold weather. Back then the fashion was fit silhouette, kneelength, and fur or silk sash-beset neckband, cuff, and hem.

Fashion overcoats in the 1920s could be divided into two kinds, dark and light color ones. The light ones were generally assorted with light ermine collar, while dark ones were matched with dark sherpa collar for the sake of harmony. But sometimes dark overcoats were beset with light fur to achieve contrastive effect.

For example, in Ladies and New Winter Clothes from Issue 34 of Professional Journal Collection in 1929, Liu Guang recorded the a fashion overcoat for ladies in Shanghai that year, which featured rectangle neckband, woolen fabric as the best, and dark color as the best. As to the tailoring, "the neckband and the shoulder should be square, other parts do not need to be beset with fur"'[1], and collar and sleeve were assorted with fur. Inside the lady wore a white cheongsam with black patterns, a pantyhose and a pare of high-heels, just like a fairy.

At the time the fabric could be either thick or thin. Most was imported, with domestic woolen fabric sometimes used.

\section{B. The 1930s}

In the 1930s, fashion overcoats were divided distinctively into overcoats for Spring, Summer, Autumn and Winter respectively. Each season's overcoats were unique in their own way in color matching, fabric, style and adornment, which vividly reflected the characteristics of the people who wore them. There were many splendid design features at the neckband, cuff, chest and shoulder. There emerged allweather overcoats with tweed on one side and cashmere on the other, overcoats using the same fabric as the cheongsam inside to form a uniform pattern, and other feature overcoats. With fashion overcoats becoming more and more popular in big cities, overcoats became usual showpieces and promotion pieces in fashion shows. 
Fashion overcoats in the 1930s featured square shoulders and wasp waist. For example, fashion winter overcoats with fur-assorted collar in 1932 were mostly square-shouldered, and were the good choice on social occasions. Besides, the partial design at this time were more and more diverse. Since 1935, broad-shoulder overcoats became fashionable, which were probably borrowed from the shoulder pad in men's suit. "This made the not so strong ladies look strong and imposing"'[2].

In the 1930s, the overcoats tended to be either waist-high or foot-long. This was largely due to the ever lengthening cheongsams matched with the overcoats, for short overcoats and long overcoats, compared with medium ones, better match with the long cheongsams and add to the holistic aesthetic feeling.

Take the short velvet overcoat as an example. Mr. Ye Qianyu, who designed a lot of fashion dresses at this time, published many drawing pictures of short overcoats, such as "Short Overcoat With Fur Collar in Late Autumn"[3], "FoxHair-Collar Short Coat"[4], "Short Coat"[5], in Linglong in 1932, which were widely received by modern ladies. Long velvet overcoat was applied. In early 1930, cheongsam came to be as long as toughing the floor, and to wear the previous overcoat would leave one-foot of the lower hem outside, which looked not good. As a result, many overcoats were lengthened, and the lower hem was even broader so as not to hinder walking.

There were also overcoats which combined China and western styles, i.e. featured the cultural element of traditional Chinese clothes and the modern western style. For example, STAR published a photo in which $\mathrm{Hu}$ Die, a famous movie star, wore a fashion overcoat, which had a fastener of the traditional knotting, with collar and cuff showing the Chinese symmetric beauty, which was a good selection combining the Chinese and western features 4.

Fashion overcoat no longer focused on the thick, warm pattern. Instead, it stressed fit-cutting, wasp waist and narrow style. The draping cutting was widely popular, and darts were made at back, chest, and waist to make the coat fitter and prettier. According to the historical records in 1933, a big pleat was made at the back, one at each side of the chest, one at each side of the lower hem of a fashion overcoat. So the coat was comfortable to wear.

The fashion was to adorn fur at neckband, lower hem and cuff in the winter coat. For example, in 1935, the fashion furadorned-collar overcoats were black woolen sheep-fox-collar satin coat, brown woolen sheep otter fur satin coat, Zhenmolong leather collar satin coat, Apaosheng leather collar sation coat, Sikangke leather collar satin coat, Llama hair collar satin coat" and so on.

Overcoats at this time were rich in hues, brightness and saturation. A fabric could have as many as tens of colors. For example, Huiluo Company once advertised for an affordable, exquisite, all-wool plain coat. According to this commercial, there were 22 colors for this wool, such as "red, purple, bright red, sky blue, light blue, dark brown, light brown, dark green, light green, jade green, black, white, dark blue, etc"[6].
Fabrics at this time were diverse. The British one was of the best quality among imported ones. Woolen, wick, plaid were quite popular. For example, red brown plaid was most popular in Spring 1937, and the historical record read that "the most fashionable Spring overcoat should apply plaid", "it was very popular among Shanghai young ladies. Everywhere we see chic girls draping plaid coats. The color was mostly red brown, long draping to the bottom, with an ironed flat bowknot at the collar'[7].

\section{The 1940s}

Different from the slim waist and square shoulder design in the 1930s, square fit featuring broad shoulders, broad sleeves and broad waistline was popular in the 1940s. For example, the loose overcoat, popular in 1946, was three times the size of a slim woman. In 1947, "the fashion tended to be loose sleeves and waist, with three or four waves at the back", "or even four or five waves for tall people, overshadowing the pattern at the front"[8].

People then noted that "I always believe that slopping shoulders, slightly-bent waist was the standard beauty. Though time advances, the criteria for beauty have reason to exist. We should not blatantly oppose shoulder padding", but "the most daunting defect is that with padded cheongsam, short coat and long overcoat, the shoulders shoulder unbearable cottons, making the lady look as strong as a big man" 5 . Therefore people held different views to this kind of coat.

In the 1940s, there were so many varieties of long, medium and short coat, together with a medium long style, i.e. neither too long nor too short. The historical record noted that "the long coat was shortened to the most and the short ones were lengthened to the most, making a mere difference of several inches between long coat and short coat"[9]. The long coat then was about 40 inches while the short 32 .

In the 1940s, the partial design of fashion coat was very variable. Common fashion elements like puff sleeve, shrot point collar were skillfully applied. Take the sleeve as an example. Various sleeve types were available then, such as horse-hoof-shaped cuff, puff sleeve, gigot sleeve, jacket sleeve, flare sleeve. Fashion in Spring, an article in "Saturday" by Mr. Jin Taijun from Hongxiang Fashion Co. noted that "there are more varieties of sleeves, such as ballshape, umbrella-shape, melon-shape and lantern-shape. Sleeve cuffs are in horse-hoof-shape, herringbone, crown, tie-style, and pleat are out, in, or three fold. The novel raglan sleeve was liked by the chic ladies. To adorn fur from silver fox, red fox and leopard at the cuff, and neckline of the padded coat was also fashionable.

Many fashion coats then modeled the style after European and American ones, or learned from the fashion books and magazines of those countries, making the styles more western-look. Besides, mandarin fashion coats were also fashionable, such as the cheongsam coat in Shanghai in 1947. This coat, a frock actually, was like the man's mandarin coat. It came into fashion because middle and low class dancing girls in Shanghai, who could not afford expensive leather wear, wore it for pretty outlook and 
coldness-protection in winter, which was then popular among all people. Melton, the cheapest among the American woolen, was widely used for making this kind of coat.

Fit in China at that time was different from that in Europe and America. Fashion designers and makers contributed a lot to the fit design. They made customized coats for women, and reformed the European and American styles based on the temperament, aesthetics and figures of Chinese women. "The made-in-American coat would make Chinese women look strange, for the loose size may hold at least two fatties"[10].

Fashionable colors were applied skillfully in coat design. There were many vivid fashion colors and were mostly influenced by those in Europe and America. Examples were the dark brown used in 1946 and the silver grey used in Spring 1947, which were light colors of high brightness and low-saturation. Other fashionable colors were moonlight, light brown, light pink, light blue, jade green, beige, and sea color, especially light brown and beige.

Many fashion fabrics for coat in the 1940s were domestic-made. Woolen fabric made in Britain was of the best and very expensive of all fabrics at that time, and was mostly used for premium fashion coats. Fabrics made in America were mostly of inferior quality. Some were as tough as rug, some thin as gauze, and were dyed with colors that did not meet Chinese people's need. So they were used mainly for ordinary coat of ordinary quality.

Tailors for women's wear and fashion design artists emerged in ROC time and the manufacturing of coat was more and more professional and exquisite. For example, fashion companies manufacturing and selling fashion coats had already been industrialized and localized in Shanghai, which was recorded clearly in the archives for fashion industry in Shanghai in ROC time.

According to Payroll No. 10 of Guild of Fashion Shops in Shanghai 1 in the Shanghai Archives, fashion coat manufacturing in the 1940s was mainly concentrated in three parts, i.e. Jing'an Temple Road \& Nanjing Road, Linshen Road \& Zhengyang Road \& Hongkou District, and Huzhou Road \& Hubei Road, ranking from high to low in production price. Woolen coats at that time were mainly jackets, plush coats, tight-waist coats, loose-waist coats, among others. Fashion manufacturing workers earned more for producing tight ones than loose ones, plus a basic salary.

\section{CONCLUSION}

Fashion overcoat, a kind of fashion clothes to prevent against coldness in ROC time, matched well with cheongsam and other fashion dress of that time. As a reflection of localization of Western fashion dress in China, it combined the features of both the West and China, and fit the figure of Chinese women well. The fashion trend of fashion overcoat in ROC was almost the same as that in the West.

In ROC time, fashion coat in Shanghai led the fashion trend in big cities in China. There appeared professional fashion coat design and production groups, and the production in Shanghai was industrialized and localized, which provided strong support for the popularity of fashion overcoats in the whole country.

\section{REFERENCES}

[1] Liu Guang. Ladies and New Winter Clothes, Professional Journal Collection.1929:34, 1.

[2] Yan Guoxiu. Talk From the Broad Shoulder Coat for Ladies This Year. 1935:21,1。

[3] Ye Qianyu. Short Overcoat With Fur Collar in Late Autumn, Linglong. 1932:70,932.

[4] Ye Qianyu. Fox-Hair-Collar Short Coat, Linglong.1932:75,1172.

[5] Ye Qianyu. Short Coat, Linglong.1932:68,840.

[6] 22 Plain Colors Coat Woolen, Shun Pao, 1933-11-5.

[7] Jiao Shijie. Red Paid Coat, Shun Pao, 1937-5-17.

[8] Jin Taijun. Fashion in Spring, Saturday.1947:775,7.

[9] Dai. Coat This Year, Saturday.1947:775,10. 